\title{
Erratum \\ TIRCAM:A MID-INFRARED CAMERA FOR GROUND-BASED ASTRONOMY
}

\author{
P.PERSI, M.FERRARI-TONIOLO and A.R.MARENZI \\ Istituto Astrofisica Spaziale, CNR, CP.67,00044 Frascati,Italy \\ M.BUSSO, L.CORCIONE, A.FERRARI, M.GAI, G.NICOLINI, \\ F.RACIOPPI and M.ROBBERTO \\ Osservatorio Astronomico di Torino, Pino Torinesei,Italy \\ G.BONAZZOLA \\ Dipartimento di Fisica Sperimentale, Universita' di Torino \\ K.SHIVANANDAN \\ Center for Advanced Space Sensing, NRL, Washington DC 20375, USA \\ and \\ G.TOFANI \\ CAISMI, CNR, Arcetri,Italy
}

\begin{abstract}
A mid-infrared ( $8-22 \mu \mathrm{m}$ ) camera, equipped with a Hughes hybrid bulk Si:As array detector of $10 \times 64$ pixels was developed for the $1.5 \mathrm{~m}$ Italian Infrared Telescope (TIRGO). The instrument was used for narrow- band 8-14 $\mu \mathrm{m}$ high-background astronomical observations. Here we present a short description of the camera, including its fast readout electronics and its optical design. When used at TIRGO, the camera has a pixel scale of 1.23 arcsec/pix. From the observations of infrared standard stars we derived a noise equivalent flux density (NEFD) that, for all the filters, is in the range $0.5-0.7 \mathrm{Jy} \times$ $\operatorname{arcsec}^{-2} \times \min ^{-1 / 2}$ and a point spread function (PSF) of the order of $3 \operatorname{arcsec}(F W H M)$. Mosaics of the extended sources Mars at 8.8, 9.8, and $12.5 \mu \mathrm{m}$, and of the star forming region Orion $\mathrm{BN}-\mathrm{KL}$ Nebula at $12.5 \mu \mathrm{m}$, are shown. The camera performances are also illustrated on the basis of the image of the post-AGB suurce CRL618.
\end{abstract}

Key words: Infrared array detectors - Infrared camera

\section{Introduction}

Astronomical mid infrared (hereafter mid-IR) direct imaging was obtained for the first time by Arens et al.(1984), using a small format $(16 \times 16)$ hybrid Si: $\mathrm{Ga}$ detector array. Although observations proved to be rather difficult, mainly due to the high background emitted by the telescope and by the atmosphere in the $10-20 \mu \mathrm{m}$ spectral regions, a few other cameras have been built in the last years, thanks to the availability of new array detectors providing a higher quantum efficiency and a format larger than the first one (e.g. Keto et al.1991; Odenwald et al.1992; Gezari et al.1992; Hora, et al.1993; Cameron et al.1993; Lagage et al.1993).

The scientific motivations for developing this kind of instruments are obviously very strong, since radiation at mid-IR wavelengths carries a large part of the information from cool matter in stars and in the diffuse interstellar 